The AstrophysicAL JouRNAL, 533:869-883, 2000 April 20

(C) 2000. The American Astronomical Society. All rights reserved. Printed in U.S.A.

\title{
EVIDENCE FOR THE HIERARCHICAL FORMATION OF THE GALACTIC SPHEROID
}

\author{
PATRICK CôTÉ ${ }^{1}$ \\ California Institute of Technology, Mail Stop 105-24, Pasadena, CA 91125 \\ RONALD O. MARZKE ${ }^{2}$ \\ Observatories of the Carnegie Institute of Washington, 813 Santa Barbara Street, Pasadena, CA 91101 \\ Michael J. WeST \\ Department of Astronomy and Physics, Saint Mary's University, Halifax, NS B3H 3C3, Canada; and Department of Physics and Astronomy, \\ University of Hawaii, Hilo, HI 96720 \\ AND \\ DANTE MinNiti \\ Lawrence Livermore National Laboratory, Livermore, CA 94550; and Department of Astronomy and Astrophysics, \\ Pontificia Universidad Católica de Chile, Casilla 306, Santiago 22, Chile \\ Received 1999 September 17; accepted 1999 November 23
}

\begin{abstract}
The possibility that the Galactic spheroid was assembled from isolated, chemically distinct protoGalactic fragments is investigated using a Monte Carlo technique designed to simulate the chemical evolution of the Galaxy in hierarchical formation scenarios which involve no gas dissipation. By comparing the observed and simulated metallicity distributions of Galactic globular clusters and halo field stars, we estimate the level of fragmentation in the collapsing proto-Galaxy. Although the formation process is highly stochastic, the simulations frequently show good agreement with the observed metallicity distributions, provided the luminosity function of proto-Galactic fragments had the form $d N \propto L^{\alpha} d L$, where $\alpha \sim-2$. While this steep slope is strongly at odds with the presently observed luminosity function of the Local Group, it is in close agreement with the predictions of semianalytic and numerical models of hierarchical galaxy formation. We discuss a number of possible explanations for this discrepancy. These simulations suggest that the Galactic halo and its globular cluster system were assembled via the accretion and disruption of $\sim 10^{3}$ metal-poor, proto-Galactic fragments by the dominant building block: a protobulge whose own metal-rich globular clusters system has been preferentially eroded by dynamical processes. This formation scenario may provide a simple explanation for the different shapes of the Galactic globular cluster and halo star metallicity distributions. Based on the similar properties of globular clusters belonging to spiral and giant elliptical galaxies, we argue that the same process (e.g., hierarchical growth involving little gas dissipation) is responsible for the formation of both giant elliptical galaxies and the bulge-halo components of spiral galaxies.
\end{abstract}

Subject headings: galaxies: halos - galaxies: spiral — Galaxy: evolution — Galaxy: halo Galaxy: structure - globular clusters: general

\section{INTRODUCTION}

It is generally believed that galaxy formation begins with the collapse of gravitationally unstable, primordial density fluctuations (e.g., Gunn \& Gott 1972; Press \& Schechter 1974). In hierarchical formation models, such as those involving cold dark matter, large galaxies then grow at the expense of their smaller counterparts (e.g., White \& Rees 1978; Blumenthal et al. 1984; Kauffmann, White, \& Guiderdoni 1993; Cole et al. 1994). Despite the impressive successes of these models, particularly in describing the clustering properties of massive galaxies (Baugh et al. 1998), they have difficulty reproducing the luminosity distribution of nearby galaxies. For instance, a generic prediction of such hierarchical models is the existence of a large population of low-mass, dark halos in the local universe (Kauffmann et al. 1993; Klypin et al. 1999). In the specific case of the Local Group, the expected number of dark halos having circular velocities $v_{c} \lesssim 30 \mathrm{~km} \mathrm{~s}^{-1}$ exceeds the observed number of faint galaxies by an order of magnitude or more (Klypin et al. 1999; Moore et al. 1999).

\footnotetext{
${ }^{1}$ Sherman M. Fairchild Fellow.

${ }^{2}$ Hubble Fellow.
}

From an empirical perspective, hierarchical models were anticipated by Searle \& Zinn (1978), who suggested that the Galactic halo formed via the protracted infall of "transient proto-Galactic fragments." As supporting evidence, they cited the lack of an abundance gradient among the outer halo globular clusters and the possible spread in age suggested by the diversity of their horizontal-branch morphologies. The extent to which this scenario differs from that of Eggen, Lynden-Bell, \& Sandage (1962), who had argued for the rapid collapse of a homogeneous proto-Galactic gas cloud, is primarily a matter of the formation timescale and the properties of the "transient proto-Galactic fragments" (particularly their masses and total numbers) since gravitational and thermal instabilities in the collapsing gas cloud must invariably lead to fragmentation into isolated starforming regions (Fall \& Rees 1985; Sandage 1990). Clearly, the key to distinguishing between these different scenarios lies in the duration of the formation process and level of fragmentation in the collapsing proto-Galaxy.

In recent years, support has grown for the notion that the halo was, at least in part, assembled from small, protoGalactic fragments as suggested by Searle \& Zinn (1978). Such evidence includes the discovery of the tidally disturbed Sagittarius dwarf galaxy (Ibata, Gilmore, \& Irwin 1994), 
numerous reports of kinematic substructure among halo field stars (Preston, Beers, \& Schectman 1994; Majewski, Munn, \& Hawley 1996; Kinman et al. 1996; Chen 1998), improved evidence for age spreads among the halo field and cluster populations (Laird \& Sneden 1996; Sarajedini, Chaboyer, \& Demarque 1997; Hesser et al. 1998), and chemical evidence for accreted substructure among halo field stars (Carney 1996; King 1997). Mateo (1996) explored the possibility that the entire Galactic halo was formed through the accretion and disruption of faint $\mathrm{dSph}$ galaxies, assumed to represent the remains of surviving "Searle-Zinn fragments." Based on a comparison of the stellar populations, dark matter, variable stars, and globular clusters of the Galactic halo with those of its present retinue of $\mathrm{dSph}$ galaxies, Mateo (1996) concluded that such a scenario is indeed plausible, provided that the bulk of the accretion occurred at early times (see also Unavane, Wyse, \& Gilmore 1996). By contrast, van den Bergh (1996) has reviewed the evidence for a rapid, and early, collapse of a single proto-Galactic cloud in the inner regions of the Milky Way.

An associated, but as yet unanswered, question is how the formation of the Milky Way and other spiral galaxies is related to that of giant elliptical galaxies. Although it is often assumed that variations in the star formation rate and gas cooling efficiency can produce end products which have markedly different morphologies (e.g., Steinmetz \& Müller 1995; Haehnelt, Steinmetz, \& Rauch 1998), there are some intriguing similarities between the Galactic globular clusters system and those of giant elliptical galaxies which suggest a closely related formation history. Most notably, many giant elliptical galaxies contain, as does the Milky Way, chemically distinct globular clusters systems (e.g., Whitmore et al. 1995; Geisler, Lee, \& Kim 1996; Forbes, Brodie, \& Grillmair 1997; Morgan 1959; Kinman 1959; Zinn 1985). Côté, Marzke, \& West (1998) showed that such multimodal globular clusters metallicity distributions may be a signature of the formation of giant elliptical galaxies through the accretion of numerous faint dwarf galaxies and/or proto-Galactic fragments. This scenario is reminiscent of the Searle \& Zinn (1978) model for the formation of the Galactic halo and the suggestion by Harris \& Pudritz (1994) that supergiant molecular clouds having masses similar to dwarf galaxies were the sites of globular cluster formation in the early universe.

In this paper, we review and compare the properties of the globular clusters associated with giant elliptical and spiral galaxies, paying particularly close attention to the globular cluster system of the Milky Way. We then describe a technique to test the possibility that the Galactic halo formed via the accretion and disruption of numerous protoGalactic fragments.

\section{A COMPARISON OF GLOBULAR CLUSTER SYSTEMS IN ELLIPTICAL AND SPIRAL GALAXIES}

Renzini (2000) argued that there exists a fundamental connection between elliptical galaxies and the bulge-halo components of spiral galaxies, suggesting that the former can be thought of as spiral bulge-halo systems which "for some reason missed the opportunity to acquire or maintain a prominent disk." Much evidence now supports this view, at least for ellipticals of intermediate luminosity: e.g., both classes obey the same $\mathrm{Mg}_{2}-\sigma$ relationship (Jablonka, Martin, \& Arimoto 1996), structural parameter relations
(Kormendy 1985; Bender, Burstein, \& Faber 1992), and $L \propto \sigma^{n}$ relation (Kormendy \& Illingworth 1983).

In this section, we review briefly the properties of globular clusters systems in spiral and elliptical galaxies, paying particular attention to their specific frequencies, metallicity distributions, and spatial distribution. In agreement with the conclusions of the above studies, we find evidence for a close connection between elliptical galaxies and the bulgehalo components of spiral galaxies. In what follows, we refer to the combined halo-bulge components of spiral galaxies as their spheroids.

\subsection{Specific Frequencies}

Harris \& van den Bergh (1981) defined the total number of globular clusters per unit host galaxy luminosity as the globular cluster specific frequency,

$$
S_{n}=N_{\mathrm{GC}} \times 10^{0.4\left(M_{V}+15\right)} .
$$

According to Harris (1991), $S_{n} \simeq 4-6$ for dwarf and giant ellipticals in rich clusters, whereas such galaxies in loose groups have $S_{n} \simeq 2-3$. By contrast, $S_{n} \simeq 1$ for spiral galaxies. This difference forms the basis of the familiar argument that giant elliptical galaxies cannot form via spiral-spiral mergers (van den Bergh 1982; cf. Ashman \& Zepf 1992).

The above value of $S_{n} \simeq 1$ for spiral galaxies is based on their total (i.e., spheroid and disk) luminosities. Since we are interested in the relative number of globular clusters per unit spheroid luminosity, the contribution of the disk to the overall luminosity of the spiral should be removed, as originally suggested by Harris (1981). From the catalog of W. E. Harris (Globular Cluster Systems in Other Galaxies 1996, hereafter GC Catalog), ${ }^{3}$ we have selected all giant elliptical and spiral galaxies having measured specific frequencies. Two spirals studied recently by Kissler-Patig et al. (1999) have also been included. Only spirals having Hubble types between Sa and Sc have been considered, since the difficulties involved in deriving spheroid luminosities for later Hubble types become severe.

For each spiral, we calculate the specific frequency by using the total number of globular clusters taken directly from the GC Catalog or Kissler-Patig et al. (1999). The spheroid luminosities have been calculated using the "bulge-to-disk" ratios given in the literature. For those galaxies lacking published bulge-disk decompositions, the mean ratio for the appropriate Hubble type given in Simien $\&$ de Vaucouleurs (1985) was used instead. For the 11 spirals, which span the range Sa to Sc, we find a mean specific frequency of $\overline{S_{n}}=3.8 \pm 2.9$ measured with respect to their spheroids.

The mean specific frequency of giant elliptical galaxies in the GC Catalog is $\overline{S_{n}}=5.2 \pm 3.2$. This value is indistinguishable from that given above for spiral spheroids, although is probably an overestimate since it includes a small number of "high- $S_{n}$ " galaxies located in the cores of the Virgo and Fornax clusters. The spirals, by contrast, are almost invariably located in loose groups. In order to gauge the possible importance of local environment, we have calculated for each object the local galaxy density, $\rho_{0}$, using the number of galaxies brighter than $M_{B}=-16$ contained within a shell of radius $0.5 \mathrm{Mpc}$ using the Nearby Galaxy Catalog of Tully (1988). The 29 giant elliptical galaxies have

\footnotetext{
${ }^{3}$ Globular Cluster Systems in Other Galaxies, compiled by W. E. Harris, 1996 March (http://www.physics.mcmaster.ca/GC/s_n.dat).
} 
$0.1 \lesssim \rho_{0} \lesssim 9.2 \mathrm{Mpc}^{-3}$, whereas the 11 spiral galaxies span the range $0.0 \lesssim \rho_{0} \lesssim 3.7 \mathrm{Mpc}^{-3}$. A total of 17 giant elliptical galaxies have $\rho_{0} \lesssim 3.7$ and are thus located in environments of comparable density. The mean specific frequency of these galaxies is $\overline{S_{n}}=3.9 \pm 2.5$, which is indistinguishable from that found for the spiral spheroids.

\subsection{Metallicity Distributions}

The dramatic recent increase in the number of galaxies having accurately measured globular cluster metallicity distributions is due primarily to the use of metallicity sensitive color indices (e.g., Ostrov, Geisler, \& Forte 1993) and the excellent imaging capabilities of the Hubble Space Telescope (HST) (e.g., Neilsen \& Tsvatanov 1999; Gebhardt \& Kissler-Patig 1999; Kundu 1999). These studies have demonstrated that the majority of giant elliptical galaxies show evidence for the presence of multiple, chemically distinct globular cluster populations.

This multimodality is not unique to giant elliptical galaxies: the Galactic globular cluster system has long been known to show a bimodal distribution in metallicity, showing distinct peaks at $[\mathrm{Fe} / \mathrm{H}] \simeq-1.6$ and -0.6 (Morgan 1959; Kinman 1959; Zinn 1985). Analysis of the globular cluster system associated with M31 suggests that it too appears significantly multimodal (Ashman \& Bird 1993; Barmby et al. 2000). Unfortunately, the sample of spiral galaxies having accurate globular cluster metallicity distributions is limited to just these two objects, although recent work has demonstrated that bimodal globular cluster metallicity distributions are observed in S0 galaxies as well (Kissler-Patig et al. 1997b; Kundu \& Whitmore 1998). At present, the only galaxies which show no evidence of multimodal globular cluster metallicity distributions are $\mathrm{dE} / \mathrm{dSph}$ galaxies of low and intermediate luminosity (i.e., $\left.M_{V} \gtrsim-17\right)$. Such objects contain only metal-poor clusters (see Fig. 1 of Côté et al. 1998).

\subsection{Spatial Distributions}

All globular cluster systems studied to date have radial profiles which are similar to, or shallower than, that of the underlying galaxy light (Harris 1986; Kissler-Patig 1997; Durrell et al. 1996). As Harris (1991) points out, no case has been found in which the globular cluster system is more centrally concentrated than the galaxy itself.

The well-studied Virgo giant elliptical galaxies M49 and M87 are known to contain two chemically distinct globular cluster populations; in both cases, the metal-rich clusters appear to be more centrally concentrated than their metalpoor counterparts (Geisler et al. 1996; Neilsen 1999; Lee, Kim, \& Geisler 1998). In the case of M49, Lee et al. (1998) report a $6 \sigma$ difference in the measured density profile slopes for the metal-rich and metal-poor globular clusters (although see McLaughlin 1999 for a dissenting view). In addition, Lee et al. (1998) note that the metal-rich clusters trace the underlying galaxy light in both radial profile and ellipticity, whereas the metal-poor clusters comprise a more extended and spherically distributed population.

At the present time, the only spiral galaxy which has been studied in sufficient detail that it is possible to investigate reliably the separate distributions of metal-rich and metalpoor clusters is the Milky Way. The Galactic globular cluster system as a whole is known to obey a threedimensional distribution of the form $\rho_{h} \sim R_{\mathrm{G}}^{-m_{h}}$, where $m_{h} \simeq 3.5$ (Harris 1976). This is in close agreement with the values of $m_{h} \simeq 3.0-3.5$ derived from halo RR Lyrae and blue horizontal-branch stars (Saha 1985; Preston, Schectman, \& Beers 1991). Harris (1976) noted, however, that no metal-rich clusters are found beyond $R_{\mathrm{G}} \sim 7 \mathrm{kpc}$ and suggested that most of these clusters are associated with the central bulge of the Galaxy, consistent with the findings of several recent studies (Minniti 1995; Barbuy, Bica, \& Ortolani 1998; Côté 1999; cf. Zinn 1985). For comparison, the density profile of the Galactic bulge is roughly $\rho_{b} \sim$ $R_{\mathrm{G}}^{-m_{b}}$, where $3.65 \lesssim m \lesssim 4.2$ (Terndrup 1988; Blanco \& Terndrup 1989). Moreover, the mean Galactocentric radius of the metal-rich cluster sample discussed by Côte (1999) is $\overline{R_{\mathrm{G}}}=3.2 \pm 2.0 \mathrm{kpc}$, which is similar to the effective radius of the Galactic bulge $\left(R_{e}=2.7 \mathrm{kpc}\right.$; Gilmore, King, \& van der Kruit 1990).

In summary, the Galactic globular cluster system, like those of all giant elliptical galaxies studied to date, has a spatial distributions which is comparable to, or slightly more extended than, that of the underlying spheroid light. Moreover, the metal-rich globular clusters are more centrally concentrated than their metal-poor counterparts.

\section{A HIERARCHICAL MODEL FOR THE FORMATION OF THE GALACTIC SPHEROID}

Given these similarities, it is natural to ask if a single model can explain the observed properties of globular clusters in both giant elliptical and spiral galaxies. For instance, models which seek to explain the formation of giant elliptical galaxies and their associated systems of metal-rich globular clusters in spiral-spiral mergers (e.g., Schwiezer 1986; Ashman \& Zepf 1992) suffer from the obvious difficulty that the spirals themselves appear to show multimodal globular cluster metallicity distributions.

In the hierarchical picture of Côté et al. (1998), the metalrich globular clusters in giant elliptical galaxies represent the clusters initially associated with the most massive protoGalactic fragment. By contrast, the metal-poor globular clusters now associated with the giant elliptical galaxy are identified as those which have been acquired during the accretion and disruption of numerous dwarf galaxies and proto-Galactic fragments (i.e., faint systems which are known to contain only metal-poor clusters). The relative number of metal-rich and metal-poor globular clusters in the giant elliptical galaxy is then assumed to reflect the luminosities (or, alternatively, masses) of the dominant proto-Galactic building block and the accreted population of smaller proto-Galactic fragments. It is important to note that this procedure neglects possible differences in the destruction rates of metal-rich and metal-poor clusters (see $\S 3.6)$. Note that both the total number of clusters and the globular cluster specific frequency are conserved during the mergers (i.e., we assume that no new clusters are formed in the merger process).

Thus, given three ingredients, it is possible to simulate the evolution of the globular cluster metallicity distribution of a specific galaxy: (1) the luminosity (or mass) function of galaxies and proto-Galactic fragments, (2) the number of globular clusters per unit fragment luminosity (or mass), and (3) the dependence of mean globular clusters metallicity on fragment luminosity (or mass). In the present case, we have an additional constraint on the formation of the Galactic spheroid: the metallicity distribution of individual halo field stars. Specifically, any acceptable simulation of 
the formation of the Galactic spheroid must reproduce not only the observed metallicity distribution of globular clusters (particularly, the two distinct peaks) but also that of the halo field stars. This latter distribution peaks at roughly the same metallicity as the metal-poor globular clusters and yet includes extended metal-poor and metal-rich tails which are not evident in the cluster distribution (Laird et al. 1988).

The various model inputs are discussed in detail below, where we concentrate on the specific case of the hierarchical formation of the Galactic spheroid.

\subsection{Stellar Metallicity-Luminosity Relation for Proto-Galactic Fragments}

Following Larson (1988), Zinn (1993), and Mateo (1996), we begin by assuming that the dwarf galaxy population of the Local Group can be thought of as the surviving building blocks of their parent galaxies. Since it has been known for some time that the mean stellar metallicity of galaxies depends rather sensitively on their total luminosity (e.g., Davies et al. 1987; Brodie \& Huchra 1991), we expect that the stellar metallicity-luminosity relation defined by these galaxies is a reasonable first approximation of that which would be expected for the proto-Galactic fragments at the present time. In other words, these fragments are assumed to have faded passively with time in the same manner as the presently observed dwarfs. It is, however, important to bear in mind that many Local Group dSph and dE galaxies have clearly managed to form stars at intermediate epochs; this would not be the case for any proto-Galactic fragments which were disrupted and depleted of gas at very early times.

In the upper panel of Figure 1, the filled circles show the dependence of mean stellar metallicity on galaxy luminosity for 28 nearby dSph, dE, and "dSph/dIrr transition" galaxies (Durrell et al. 1996; Mateo 1998; Caldwell et al. 1992; Côté, Oke, \& Cohen 1999). The best-fit linear relation is given by

$$
\overline{[\mathrm{Fe} / \mathrm{H}]_{*}}=-3.43( \pm 0.14)-0.157( \pm 0.012) M_{V},
$$

which implies $L \propto Z^{2.54 \pm 0.19}$. For comparison, the dotted line indicates the scaling relation, $L \propto Z^{2.7}$, which is expected for dwarf galaxies which form in standard cold

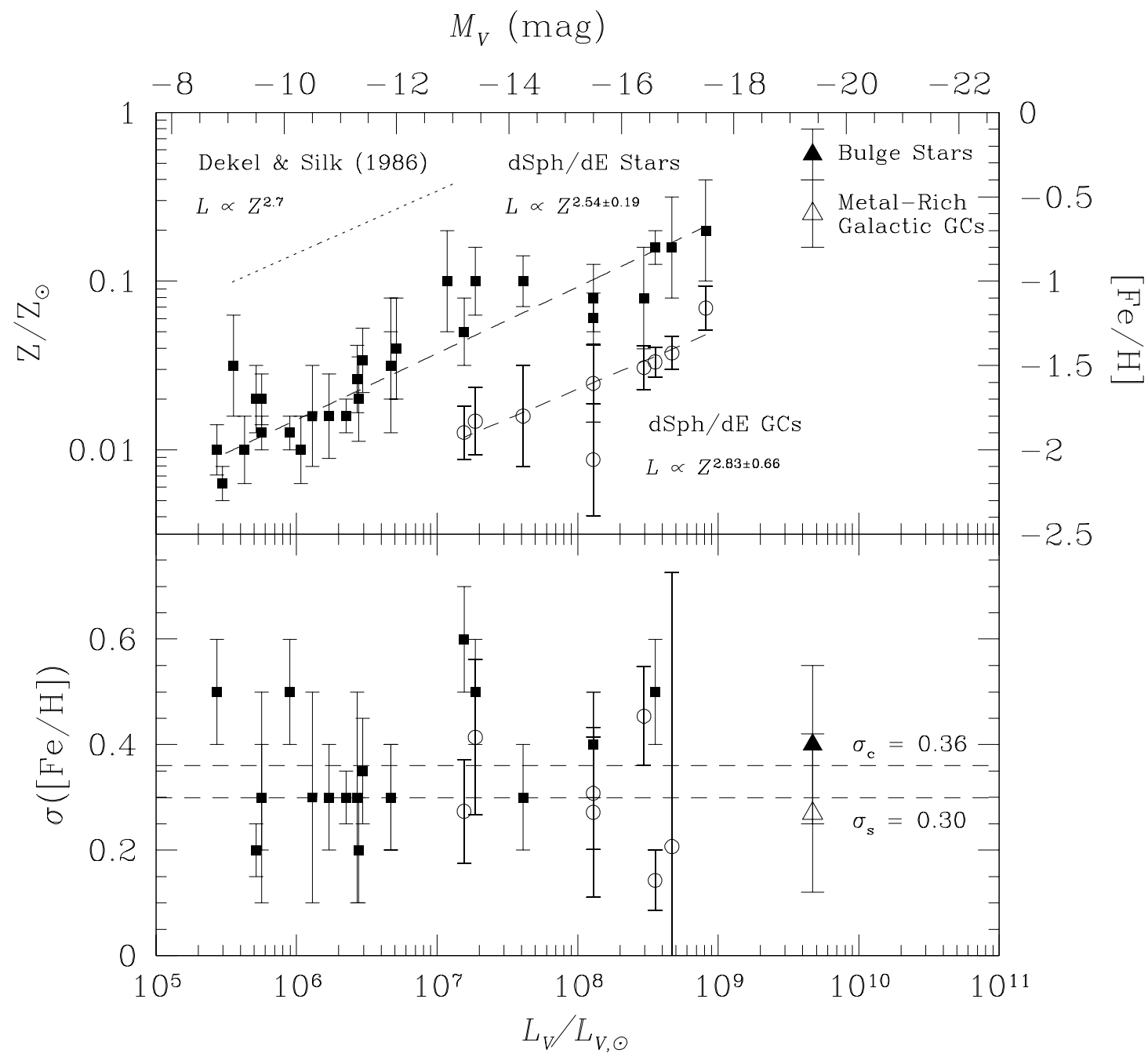

FIG. 1.-(Upper panel) Relation between total luminosity and the mean metallicity of stars ( filled squares) and globular clusters (open circles) belonging to early-type dwarf galaxies. The filled triangle indicates the mean metallicity of Galactic bulge stars; the open triangle shows the mean metallicity of metal-rich globular clusters in the Milky Way. The best-fit linear relations for the stars and clusters are indicated by the dashed lines, while the solid line indicates the predicted relation of Dekel \& Silk (1986), shifted vertically by an arbitrary amount. This theoretical relation is indistinguishable from the empirical relations observed for both the stars and globular clusters. (Lower panel) Observed dispersion in metallicity for stars (squares) and globular clusters (circles) for the same sample. The filled and open triangles indicate the observed dispersions for Galactic bulge stars and metal-rich Galactic globular clusters, respectively. 
dark matter scenarios (Dekel \& Silk 1986). Note the arbitrary metallicity zero point of the latter relation. We conclude that equation (2) is a reasonable representation of the stellar luminosity-metallicity relation of surviving protoGalactic fragments. Although it has not been included in the fit, the filled triangle in this figure indicates the location of the Galactic bulge (McWilliam \& Rich 1994). Note that its location is consistent with the extrapolation of the fitted relation for dwarf galaxies.

Although the mean stellar metallicity in dSph and $\mathrm{dE}$ galaxies depends rather sensitively on total luminosity (and, presumably, total mass), there is now unmistakable evidence for sizeable abundance spreads within individual objects. The standard deviations in $[\mathrm{Fe} / \mathrm{H}]$, assuming a Gaussian distribution of abundances, for these galaxies are shown in the lower panel of Figure 1. The data are taken mainly from the catalog of Mateo (1998) and have been supplemented with a few recent results on the M31 dSph system. The mean value of $\sigma\left([\mathrm{Fe} / \mathrm{H}]_{*}\right)=0.36 \pm 0.11 \mathrm{dex}$ is indicated by the upper dashed line. Unlike the mean metallicity, the dispersion in metallicity depends weakly, or not at all, on total luminosity (Côté et al. 1999). The possible implications of this result are discussed in the following section.

\subsection{Globular Cluster Metallicity-Luminosity Relation for Proto-Galactic Fragments}

The open circles in the upper panel of Figure 1 show the mean metallicity of globular clusters as a function of total galaxy luminosity for $\mathrm{dSph}$ and $\mathrm{dE}$ galaxies in the Local Group, M81, and Virgo (see, e.g., Côté et al. 1998 and references therein). The best-fit linear relation is given by

$$
\overline{[\mathrm{Fe} / \mathrm{H}]_{\mathrm{GC}}}=-3.79( \pm 0.53)-0.141( \pm 0.033) M_{V} \text {. }
$$

The corresponding relation, $L \propto Z^{2.83 \pm 0.66}$, is, like the stellar metallicity-luminosity relation, in excellent agreement with the predictions of Dekel \& Silk (1986). It is also indistinguishable from that implied by equation (2) with the notable exception of a $\Delta[\mathrm{Fe} / \mathrm{H}] \sim 0.6$ dex offset between the clusters and stars (in the sense that the clusters are a factor of $\sim 4$ more metal-poor). For comparison, the open triangle indicates the mean metallicity of the metal-rich Galactic globular clusters (which has not been included in the fit). The difference in metallicity between the bulge stars and metal-rich globular clusters is roughly $\Delta[\mathrm{Fe} / \mathrm{H}]=$ $0.35 \pm 0.25$ dex: i.e., smaller than, but consistent with, the metallicity offset seen in the dwarf galaxies.

The lower panel of Figure 1 shows the intrinsic dispersions in metallicity for globular clusters in these galaxies, plotted against absolute visual magnitude of the host galaxy. The mean value, $\sigma\left([\mathrm{Fe} / \mathrm{H}]_{\mathrm{GC}}\right)=0.30 \pm 0.11 \mathrm{dex}$, is indicated by the lower dashed line. To within the errors, this dispersion is the same as that of the stars and, similarly, shows no obvious trend with luminosity.

The theoretical metallicity-luminosity relation of Dekel \& Silk (1986) is based on the key assumptions that these objects originated as gaseous protogalaxies embedded in dominant dark matter halos whose chemical enrichment was dictated by enrichment from massive stars and gas loss via supernovae-driven winds. In such a scenario, the chemical evolution is approximated by the so-called Simple Model of chemical evolution (Searle \& Sargent 1972; Pagel $\&$ Patchett 1975; Hartwick 1976). The success of this model in reproducing the observed metallicity-luminosity rela- tions shown in Figure 1 suggests that it may also provide a convenient representation of the metallicity distribution internal to each proto-Galactic fragment. This success is all the more remarkable in view of the fact that many of the galaxies shown in Figure 1 exhibit incontrovertible evidence for multiple star formation bursts, whereas the Dekel \& Silk (1986) model was framed within the context of a single star formation event.

For a homogeneous proto-Galactic gas cloud having zero initial metallicity and a yield, $y$, the metallicity distribution at the end of gas exhaustion takes the form

$$
d f / d z \propto y^{-1} \exp (-z / y) .
$$

This distribution follows from the usual assumptions of the Simple Model: i.e., the initial mass function is constant in time, and the proto-Galactic cloud experiences recycling of heavy elements from massive stars whose lifetimes are short compared to the free-fall timescale of the gas cloud. In an attempt to explain the lower metallicities of halo globular clusters compared to the Galactic disk, Hartwick (1976) defined an effective yield, $y_{e}$, given by the relation $y_{e}=y /$ $(1+c)$, where $c$ is a parameter related to the rate at which gas is lost from the system (via, for example, supernovaedriven winds). Thus, in this picture, the effective yield of each fragment or gas cloud is determined by its overall mass (see, e.g., Binney \& Merrifield 1998).

The upper panel of Figure 2 shows the stellar metallicity distribution predicted by equation (4) for a galaxy having $M_{V}=-15$, where we have assumed that the effective yield is equal to the mean stellar metallicity predicted by equa-

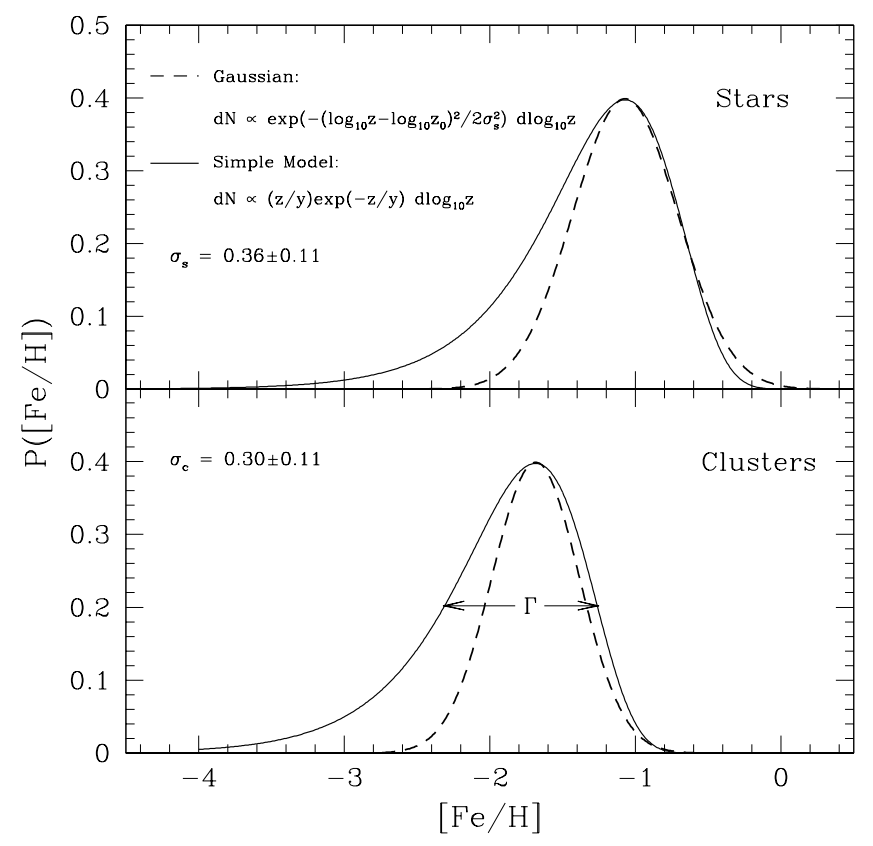

FIG. 2.-(Upper panel) Predicted internal stellar metallicity distribution according to the Simple Model for a proto-Galactic fragment having a

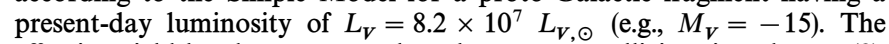
effective yield has been set equal to the mean metallicity given by eq. (2). For comparison, the dashed curve shows a Gaussian with dispersion $\sigma_{s}([\mathrm{Fe} / \mathrm{H}])=0.36$ and the same modal metallicity. (Lower panel) Predicted distribution of globular cluster metallicities based on the Simple Model for this same proto-Galactic fragment. In this case, the effective yield is equal to the mean metallicity given by eq. (3). As before, the dashed curve shows a Gaussian with dispersion $\sigma_{s}([\mathrm{Fe} / \mathrm{H}])=0.30$ and the same modal metallicity. 
tion (2). ${ }^{4}$ The lower panel shows the expected distribution for globular clusters assuming that the effective yield is given by equation (3). These distributions have, of course, identical shapes and FWHMs $(\Gamma \simeq 1.0$ dex, corresponding to $\sigma \sim 0.44 \mathrm{dex}$ ), but they are offset by $\Delta[\mathrm{Fe} / \mathrm{H}] \simeq 0.6 \mathrm{dex}$. Based on the success of the Dekel \& Silk (1986) metallicityluminosity relation, and the roughly constant spread in metallicity exhibited by both stars and clusters in these galaxies, we suggest that equation (4) is a reasonable first approximation of the stellar and globular cluster metallicity distributions of dwarf galaxies and proto-Galactic fragments. Refinements to the Simple Model, such as the inclusion of possible gas inflow and outflow, tend to produce narrower metallicity distributions (e.g., Gilmore et al. 1990).

\subsection{The Luminosity and Mass Functions of Proto-Galactic Fragments}

As in Côté et al. (1998), the initial Galactic luminosity function (i.e., the luminosity distribution of proto-Galactic fragments) is approximated by a Schechter function,

$$
d N \propto\left(L / L^{*}\right)^{\alpha} \exp \left(-L / L^{*}\right) d L,
$$

where $L^{*}$ is a characteristic luminosity and $\alpha$ is an exponent which governs the relative number of faint and bright systems (Schechter 1976). For early-type systems in lowdensity environments such as the Local Group, $L_{B}^{*} \simeq 8.2$ $\times 10^{9} L_{B, \odot}$ assuming $H_{0}=75 \mathrm{~km} \mathrm{~s}^{-1} \mathrm{Mpc}^{-1}$ (Marzke et al. 1998). Since our goal is to model the growth of the Galactic spheroid (which has $L_{B} \sim 3.9 \times 10^{9} L_{B, \odot}$; see $\S 3.4$ ), the above representation is effectively a power-law distribution in luminosity:

$$
d N \propto L^{\alpha} d L .
$$

This distribution is similar to the mass spectrum expected in some hierarchical cosmologies. For instance, in cold dark matter models, the index of the initial power spectrum is $-3<n<-2$ on the scales of dwarf galaxies, which leads to mass function of the form $N(M) \propto M^{-2}$ (e.g., Blanchard, Valls-Gabaud, \& Mamon 1992; Ferguson \& Bingelli 1994). Unfortunately, the transformation to a luminosity spectrum remains highly uncertain since it involves several poorly understood processes such as gas cooling, star formation, and feedback from massive stars. If the cosmological models are correct, then the fact that the galaxy luminosity function today is shallower than the primordial mass spectrum suggests that there is not a one-to-one correspondence between galaxy mass and luminosity and/or that the luminosity function has been modified over time, perhaps by mergers (see $\S 5$ ).

The maximum fragment luminosity is dictated by the requirement that the total spheroid luminosity must not exceed the observed value. The faint-end cutoff is taken to be $L_{V}=2.7 \times 10^{5} L_{V, \odot}$, the luminosity of the faintest galaxy (i.e., Draco) used to define the metallicity-luminosity relation of proto-Galactic fragments (\$3.1). Fragments having luminosities below that of the Fornax dSph galaxy (i.e., $L_{V} \leq 1.6 \times 10^{7} L_{V, \odot}$ ) are assumed to contribute no globular clusters, since this is the faintest galaxy known to contain its own globular cluster system.

\footnotetext{
${ }^{4}$ Strictly speaking, $y_{e}$ refers to the mode of the metallicity distribution given by eq. (4).
}

\subsection{Number of Globular Clusters per Proto-Galactic Fragment}

An alternative representation of equation (1) is

$$
N_{\mathrm{GC}}=\eta L_{V}
$$

where $\eta=\left(1.2 \times 10^{-8}\right) S_{n}$ clusters $L_{V}^{-1} \odot$. There is some recent evidence that specific frequency may not be a linear function of luminosity. For instance, as discussed in $\S 2.1$, most early-type giant galaxies and spiral spheroids have globular cluster specific frequencies of $S_{n} \sim 4$, whereas the dSph and dE galaxies shown in Figure 1 have a marginally higher mean specific frequency of $S_{n}=8 \pm 3$. From $H S T$ imaging of $\mathrm{dE}$ galaxies in the Virgo cluster, Miller et al. (1998) find $S_{n}=3.1 \pm 0.5$, with little or no luminosity dependence. On the other hand, they find $S_{n}=6.5 \pm 1.2$ for nucleated Virgo dE galaxies and see clear evidence of a trend for $S_{n}$ to increase with decreasing luminosity (i.e., rising from $S_{n} \sim 3$ at $M_{V} \sim-17$ to $S_{n} \sim 20$ at $M_{V} \sim$ -13.5) Based on their results, and on the $N_{\mathrm{GC}}-L_{V}$ relations for early-type dwarf and giant galaxies given in KisslerPatig et al. (1997a) and McLaughlin (1999), we express the initial number of globular clusters, $N_{\mathrm{GC}}$, associated with each proto-Galactic fragment as

$$
N_{\mathrm{GC}}=\eta L_{V}^{\beta},
$$

where $L_{V}$ is in solar units. For $L_{V} \leq 2 \times 10^{9} L_{V, \odot}$, we take $\beta=0.8$ and $\eta \simeq 5 \times 10^{-6}$. Above this luminosity, we assume $\beta=1.1$ and $\eta \simeq 6 \times 10^{-9}$.

\subsection{Adopted Luminosities for the Galactic Bulge and Halo}

By definition, the luminosity of the Galactic spheroid, $L_{V}^{s}$, is given by the combined luminosities of the Galactic halo, $L_{V}^{h}$, and bulge, $L_{V}^{b}$. De Vaucouleurs \& Pence (1978) give $L_{V}^{s}=4.7 \times 10^{9} L_{V, \odot}$ for the combined $R^{1 / 4}$ component of the Milky Way. This is considerably smaller than the value of $L_{V}^{s}=1.1 \times 10^{10} L_{V, \odot}$ found by Blanco \& Terndrup (1989). In what follows, we shall adopt $L_{V}^{s}=7.7 \times 10^{9}$ $L_{V, \odot}$, which represents the mean of these two determinations.

Unfortunately, estimates of the separate luminosities of the Galactic bulge and halo are uncertain owing to the overlapping distributions of disk, halo, and bulge stars in the inner Galaxy (see, e.g., Morrison 1996), the possible presence of a metallicity gradient in the bulge (Minniti et al. 1995), and the unknown shape of the halo density profile in the inner few kiloparsecs. In what follows, we adopt a bulge luminosity of $L_{V}^{b}=5 \times 10^{9} L_{V, \odot}$ (Dwek et al. 1995; Holtzmann et al. 1998) which, when combined with the above value of $L_{V}^{s}$, gives $L_{V}^{h}=2.7 \times 10^{9} L_{V, \odot}$. This estimate is consistent with that of Suntzeff, Kinman, \& Kraft (1991), who used the relative space densities of globular clusters and field RR Lyrae stars to derive a total halo luminosity of $5.8 \times 10^{8} L_{V, \odot}$ over the range $4 \leq R_{\mathrm{G}} \leq 25 \mathrm{kpc}$ : i.e., our halo luminosity is equivalent to theirs for inner and outer limits on the halo population of $R_{\mathrm{G}} \simeq 0.5$ and $125 \mathrm{kpc}$, respectively.

\subsection{Dynamical Evolution and Globular Cluster Destruction}

In their study of the globular cluster systems of giant elliptical galaxies, Côté et al. (1998) made the first-order assumption that the metal-rich and metal-poor clusters have suffered equal rates of destruction through dynamical processes. However, given the evidence for different spatial distributions among the metal-rich and metal-poor sub- 
systems, this assumption may be not valid since more rapid erosion is expected in the denser environments. As it seems inescapable that the Galactic globular clusters system has been depleted by dynamical processes (Ostriker, Spitzer, \& Chevalier 1972; Tremaine 1976; Fall \& Rees 1985; Aguilar, Hut, \& Ostriker 1988; Gnedin \& Ostriker 1997; Murali \& Weinberg 1997) and that the likelihood of disruption for a given globular cluster depends sensitively on its orbit, erosive effects are expected to be more severe for the centrally concentrated, metal-rich globular cluster system.

We have attempted to incorporate the effects of dynamical evolution on the simulated globular cluster metallicity distributions by adopting the results of the Fokker-Planck calculations of Murali \& Weinberg (1997). These calculations include the combined effects of relaxation, tidal heating, and binary heating. The upper panel of Figure 3 shows the initial and final cumulative radial distributions for Milky Way spheroid globular clusters taken directly from Murali \& Weinberg (1997). In the lower panel, we have plotted the ratio of the derivatives of these two curves, which we take as a rough estimate of the "survival probability," $P_{S}$, for a typical globular clusters orbiting in the Galactic potential for a Hubble time. For $R_{\mathrm{G}}>30 \mathrm{kpc}$, the upper limit on Galactocentric radius considered by Murali \& Weinberg (1997), we take the $P_{S}\left(R_{\mathrm{G}}\right) \equiv 1$. The minimum Galactocentric radius used in the simulations is $R_{\mathrm{G}}=0.8$ kpc.

The presently observed density profiles of the (collisionless) bulge and halo field star populations are then used to assign randomly an initial Galactocentric radius to each globular cluster. In other words, following Harris (1976), Minniti (1995), Barbuy, Bica, \& Ortolani (1998), and Côté (1999), we associate the bulk of the metal-rich cluster population with the Galactic bulge and not the thick disk

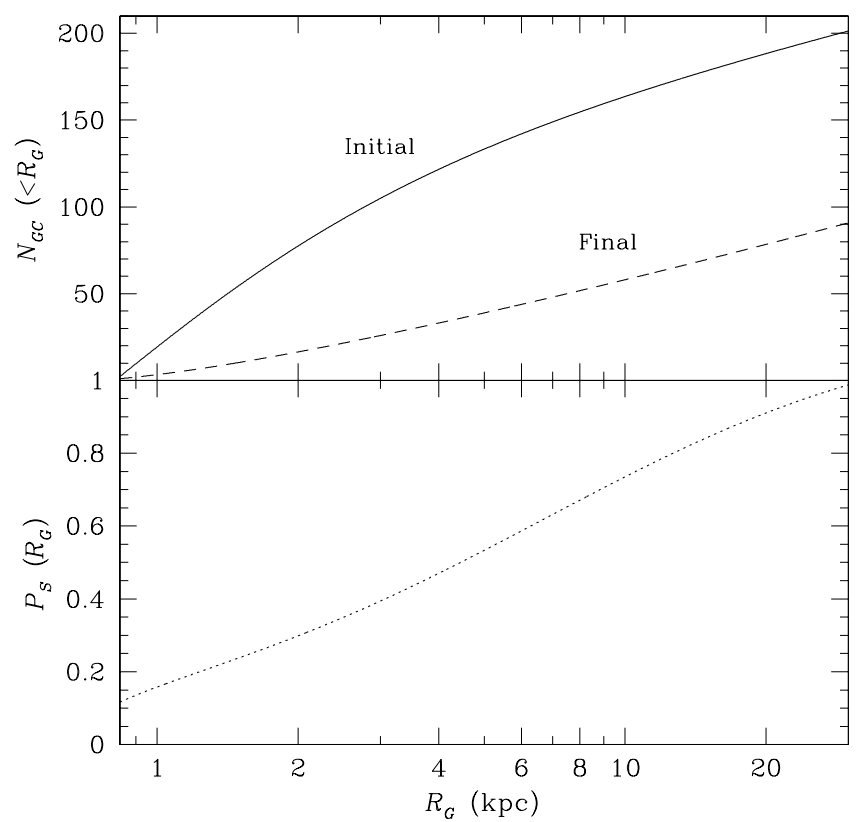

FIG. 3.-(Upper panel) Initial and final distributions for globular clusters belonging to the Galactic spheroid according to the Fokker-Planck calculations of Murali \& Weinberg (1997). (Lower panel) Survival probability as a function of Galactocentric radius for globular clusters calculated from the cumulative distributions shown above (see text for details). Clusters beyond $R_{\mathrm{G}}=30 \mathrm{kpc}$ are assumed to have survival probabilities of unity.
(Zinn 1985; Armandroff \& Zinn 1988). The Murali \& Weinberg (1997) survival probabilities are then used to decide, on a cluster-by-cluster basis, which objects should be kept in the sample and which should discarded as likely candidates for disruption. The adopted density profile for the halo is $\rho_{h}\left(R_{\mathrm{G}}\right) \propto R_{\mathrm{G}}^{-3.5}$ (Saha 1985; Preston et al. 1991), while the bulge density profile is taken to be $\rho_{b}\left(R_{\mathrm{G}}\right) \propto R_{\mathrm{G}}^{-4.0}$ (Terndrup 1988; Blanco \& Terndrup 1990; Frogel et al. 1990).

Since the calculations of Murali \& Weinberg (1997) assume a fixed Galactic potential, they may not be strictly appropriate for a model in which the Galactic spheroid is assembled from a collection of distinct proto-Galactic fragments. Nevertheless, they should at least provide a qualitative description of the dynamical evolution of the separate globular cluster systems since, in this scheme, dynamical erosion alters the overall number of metal-rich and metalpoor clusters but does not change the shape of their metallicity distributions.

\section{COMPARISON OF THE OBSERVED AND SIMULATED METALLICITY DISTRIBUTIONS}

\subsection{Methodology}

The algorithm used to generate the simulated metallicity distributions is based on that described in Côte et al. (1998). The reader is referred to that paper for a detailed discussion of the model assumptions. Here, we give only a brief description of the model as it is applied to the specific case of the Milky Way.

The first step in the simulations is to generate a metalrich system of bulge field stars and metal-rich globular clusters by combining the adopted bulge luminosity with metallicity-luminosity relations given by equations (2) and (3) and by assuming that the internal metallicity distribution of the bulge, like those all other proto-Galactic fragments, is accurately represented by equation (4). This procedure is then repeated for additional proto-Galactic fragments, each drawn at random from the luminosity distribution given by equation (5), until the combined luminosity is equal to observed luminosity of the Galactic spheroid. The number of globular clusters belonging initially to each fragment is calculated using equation (8), while the relative number of stars contributed by the various fragments are given simply by their luminosities. For globular cluster system, we approximate dynamical evolution on a cluster-by-cluster basis using the Monte Carlo approach discussed in $\S 3.6$. The simulations are performed for a wide range in the adopted power-law exponent of the proto-Galactic luminosity function. A comparison of the observed and simulated metallicity distributions for both the globular clusters and halo field stars is then used to decide which luminosity functions produce acceptable agreement. As in Côté et al. (1998), we assume that the proto-Galactic fragments have equal merger probabilities; the reader is referred to that paper for a discussion of the possible effects of dynamical friction on the simulations.

\subsection{Globular Cluster Metallicity Distributions}

Perhaps the most noteworthy feature of the simulations presented here is the diversity of the end products: the simulated globular cluster metallicity distributions show a wide range in appearance, ranging from unimodal distributions to more complex ones having multiple distinct peaks. This 
diversity is not unexpected in a stochastic process such as galaxy formation and differs from the predominantly bimodal globular cluster metallicity distributions found previously for giant elliptical galaxies (Côté et al. 1998) for two simple reasons. First, by virtue of the globular cluster metallicity-luminosity relation and the modest luminosity of the Galactic bulge, the mean metallicity of the metal-rich clusters is not as widely separated from that of the metalpoor component. Second, the high luminosities of giant elliptical galaxies permit the accretion of correspondingly more luminous proto-Galactic fragments or galaxies, meaning that the exponential cutoff in the luminosity distribution given by equation (5) imposes a sharp cutoff on the metal-rich side of the distribution of globular clusters arising in proto-Galactic fragments. Such a cutoff, which serves to delineate the globular clusters of the dominant proto-Galactic fragment from those of the other fragments, does not apply in the case of the Galactic spheroid since $L_{V}^{s} \lesssim L_{V}^{*}$, as discussed in $\S \S 3.3$ and 3.5.

The principal conclusions drawn from these simulations can be summarized as follows: (1) the bulge, as the dominant proto-Galactic building block, is observed to have the most metal-rich globular clusters system by virtue of the globular cluster metallicity-luminosity relation; (2) the bulge contributes roughly twice the number of globular clusters initially as do the combined halo progenitors; (3) the more centrally concentrated metal-rich globular cluster system has been preferentially eroded by dynamical effects; (4) the metal-poor cluster system exhibits a much wider range in its observed properties although the simulations reveal that $\alpha \sim-2$ produces the closest match to the observed distribution, particularly the peak at $[\mathrm{Fe} / \mathrm{H}] \sim$ -1.6 ; and (5) the metal-poor clusters, being more spatially extended than their metal-rich counterparts, have undergone less severe dynamical erosion.

The upper panel of Figure 4 shows a single simulation of globular cluster metallicity distribution, specifically chosen to match roughly the basic properties of the Galactic globular cluster system. The actual distribution, based on a sample of 133 clusters having measured metallicities (Harris 1996), is indicated by the open circles. The two dotted curves show the initial distributions of bulge and halo globular clusters, while the dashed curves indicate the same distributions after including the effects dynamical erosion. The spheroid in this simulation has $L_{V}^{s}=7.4 \times 10^{9} L_{V, \odot}$ and contains 149 surviving globular clusters, descended from an original population of 429. In this particular simulation, the halo was assembled from a total of 1309 proto-Galactic fragments having a combined luminosity of $L_{V}^{h}=2.7 \times 10^{9} L_{V, \odot}$. The final specific frequency of the Galactic spheroid is $S_{n}=1.6$, which is identical to the observed value.

\subsection{Halo Star Metallicity Distributions}

The solid curve in the lower panel of Figure 4 shows the halo star metallicity distribution for the simulation described above. Open circles indicate the actual metallicity distribution of 372 kinematically selected halo field stars according to Ryan \& Norris (1991). Both the simulated and observed distributions show maxima in the range $-1.7 \lesssim[\mathrm{Fe} / \mathrm{H}] \lesssim-1.5$ and broad wings extending to lower and higher metallicities. These extended tails are more pronounced in the field star distribution than in the cluster metallicity distribution. The significance of these differences has always remained somewhat questionable

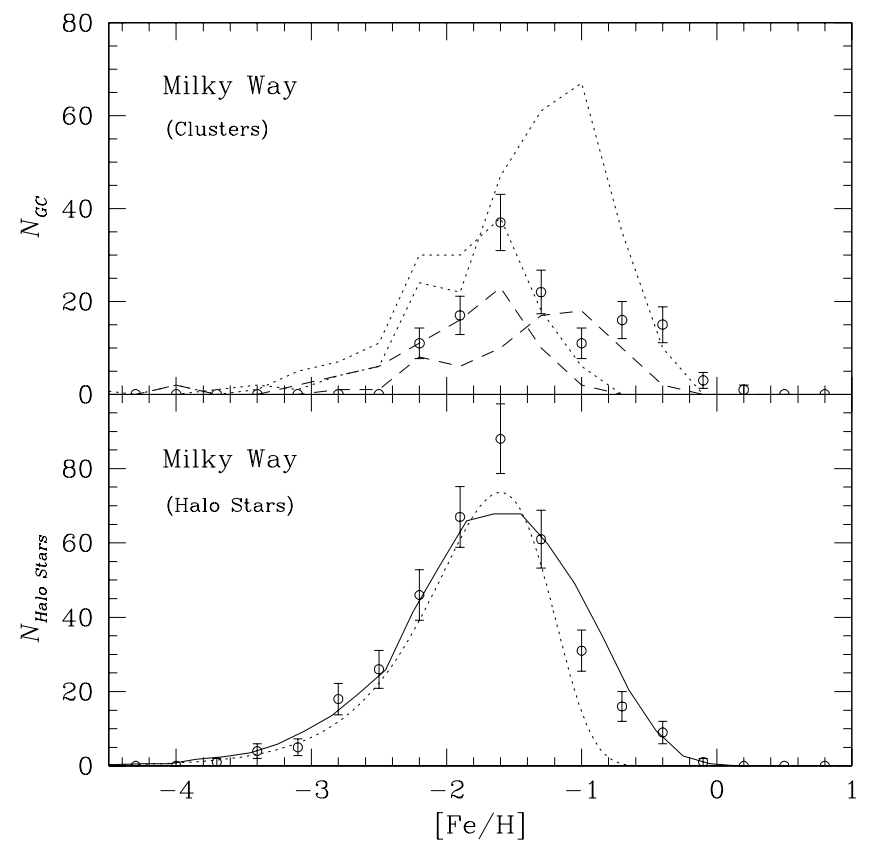

FIG. 4.-(Upper panel) One simulation of the Galactic globular cluster metallicity distribution, before (dotted curves) and after (dashed curves) the effects of dynamical evolution are included. The metal-rich and metal-poor components indicate the respective globular cluster systems of the protobulge and protohalo. The actual distribution, based on data from Harris (1996), is indicated by the open circles. (Lower panel) Corresponding distribution of halo field star metallicities based on this simulation (solid curve). The open circles show the halo metallicity distribution based on the data of Ryan \& Norris (1991). The dotted line shows the prediction of the Simple Model for an effective yield of $\log y_{e}=-1.6$.

owing to the finite size of the Galactic cluster system (i.e., the discrepancy at the metal-poor and metal-rich ends can removed by adding only four and six clusters, respectively; Laird et al. 1988).

Nevertheless, such differences are often seen in the simulations described here, and their origin can be understood as follows. The extended metal-poor tail in the field star distribution is populated exclusively by stars formed in the faintest and most metal-deficient proto-Galactic fragments: i.e., those having mean metallicities of $[\mathrm{Fe} / \mathrm{H}] \sim-2.1$ (see Figure 5). Such a tail is slightly less evident in the globular cluster distribution since only proto-Galactic fragments having $L_{V} \gtrsim 1.6 \times 10^{7} \quad L_{V, \odot}$ contribute clusters to the spheroid, limiting the mean metallicity of these clusters to $[\mathrm{Fe} / \mathrm{H}] \sim-1.9$. More significantly, the comparatively large number of faint proto-Galactic fragments incorporated into the halo ensures that the metal-poor tail of simulated halo metallicity distribution is well populated, in contrast to that of the globular cluster distribution (which, unlike the field star distribution, is further depleted by dynamical effects). At the metal-rich end, the small number of luminous proto-Galactic fragments incorporated into the spheroid contribute significant numbers of both globular clusters and field stars; as discussed in $\S 3.2$, these stars will be systematically $\sim 0.6$ dex more metal rich than the associated clusters and, consequently, will produce a metal-rich tail which will not be seen in the globular cluster metallicity distribution.

For comparison, the dotted curve in the lower panel of Figure 4 shows the prediction of the Simple Model for an effective yield of $\log _{10} y_{e}=-1.6$, an arbitrary value chosen 


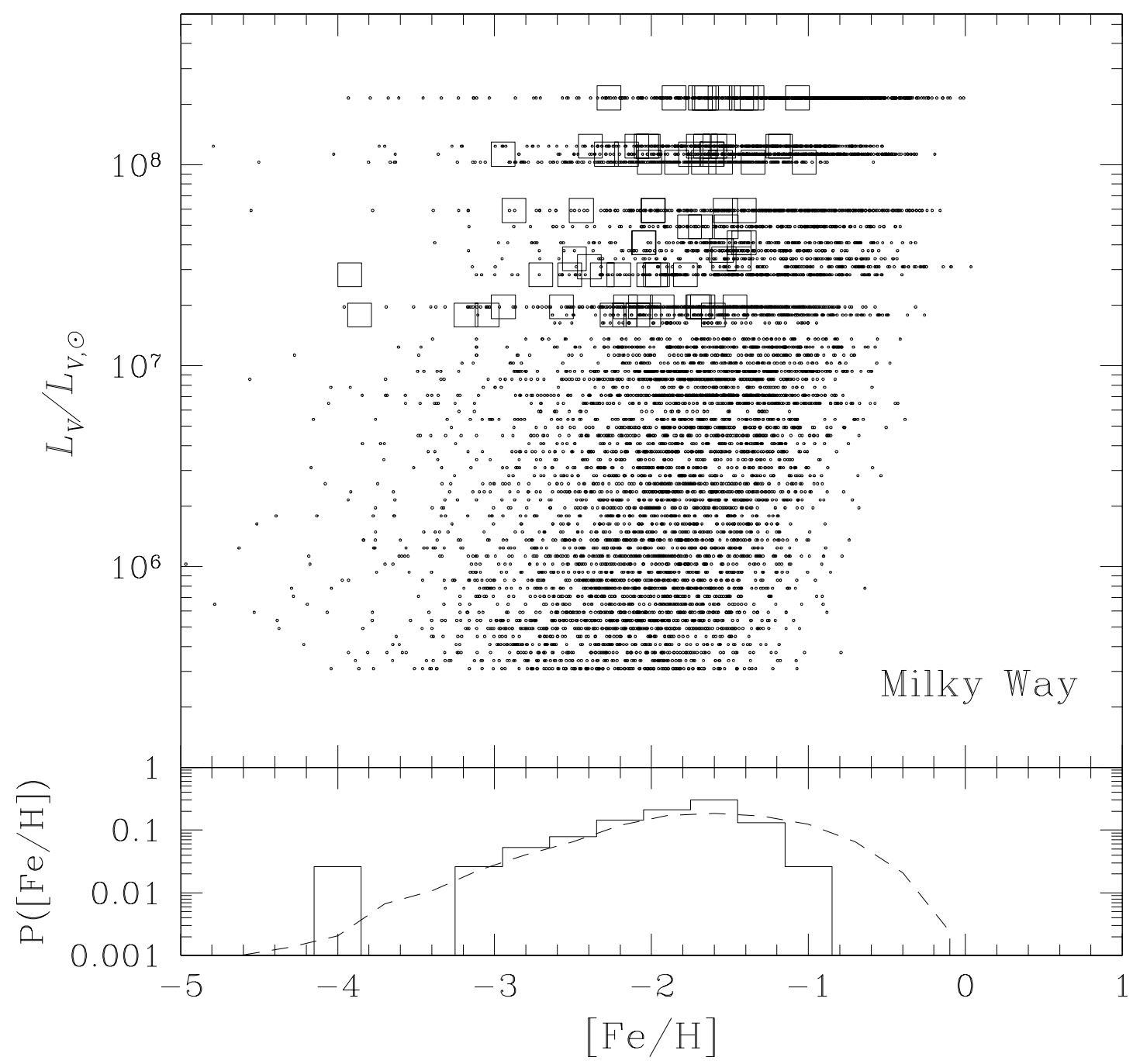

FIG. 5.-(Upper panel) Metallicities of globular clusters (squares) and halo field stars (dots) plotted as a function of the luminosity of the proto-Galactic fragment in which they originated. (Lower panel) Comparison of the metallicity distributions of globular clusters (solid curve) and halo field stars (dashed curve). Although the two samples have similar mean metallicities, the halo star population shows more extended metal-poor and metal-rich tails.

by Ryan \& Norris (1991) to give the closest match to the observed distribution. As noted by both Laird et al. (1988) and Ryan \& Norris (1991), at high metallicities the Simple Model shows poor agreement with the actual distribution. While the significance of this discrepancy is unclear owing to possible contamination by metal-rich disk stars, we note that the simulations show significant numbers of stars having $[\mathrm{Fe} / \mathrm{H}]>-1$ whose origin can be traced to the largest proto-Galactic fragments incorporated in the spheroid. The fraction of such stars in the simulations, however, is somewhat larger than that seen in the distribution of Ryan \& Norris (1991).

A consistency check on the radial distribution of the simulated globular cluster systems is shown in Figure 6. The upper and lower panels indicate histograms of Galactocentric radii for the actual and simulated globular clusters systems. In the latter case, we plot the radial profiles before and after dynamical effects. Initially, the simulated globular clusters follow a profile given by the adopted halo and bulge density laws, shown as the solid and dashed lines in the upper panel. Afterward, the surviving globular clusters have a radial distribution similar to that of the observed globular clusters, including the same flattening of the profile in the central $R_{\mathrm{G}} \sim 3-5 \mathrm{kpc}$. For the distant metal-poor globular clusters, the initial distribution is relatively unaltered.

\subsection{How Fragmented Was the Proto-Galactic Spheroid?}

Figure 7 illustrates some properties of the proto-Galactic fragments from which the Galactic spheroid was assembled. In the upper panel, we show the luminosity distribution of proto-Galactic fragments found in the above simulation. The location of the protobulge is indicated by the vertical arrow, while the dashed line indicates a power-law luminosity function having slope $\alpha=-2$. The dotted line in the lower panel shows the cumulative luminosity distribution of these same proto-Galactic fragments. Given the steep luminosity function, the vast majority of the proto-Galactic fragments are, as expected, low-luminosity systems. Unfortunately, the conversion from luminosity to mass for these fragments is highly uncertain due their unknown mass-tolight ratios. For illustrative purposes, two different mass distributions are shown in the lower panel of Figure 7. In the first case, we have assumed $M / L_{V}=2$ in solar units. This would be expected for an old stellar population whose dark matter content can be understood purely in terms of normal stellar remnants, as is the case for globular clusters 


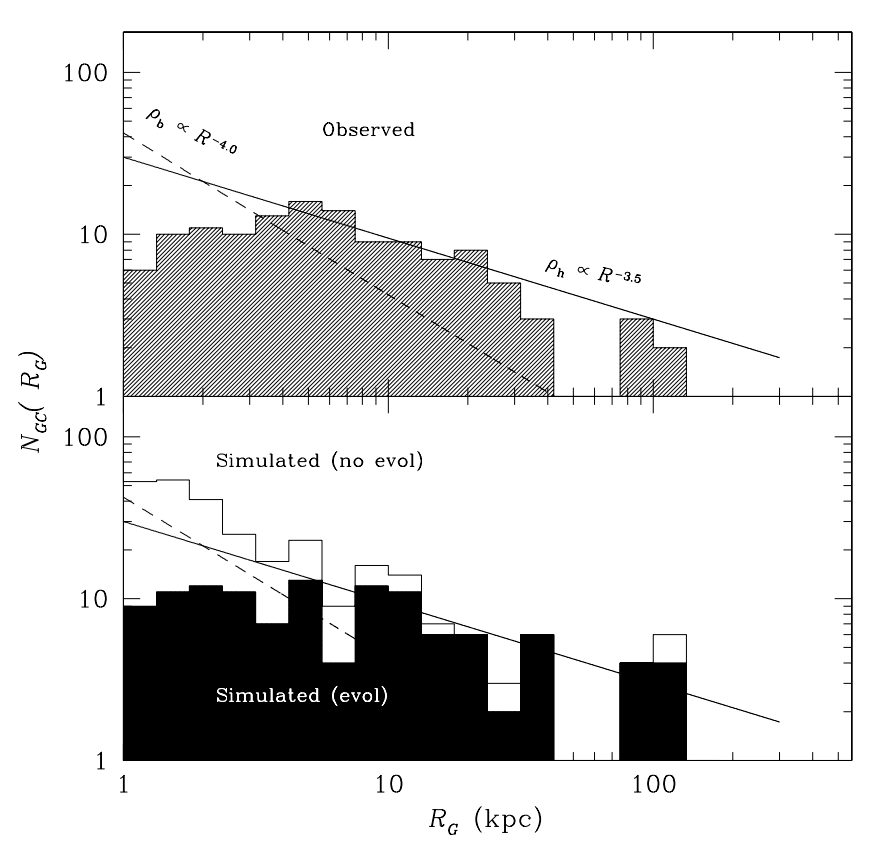

FIG. 6.-(Upper panel) Distribution of Galactocentric radii for Galactic globular clusters. The radial profiles corresponding to the density laws $\rho_{h}\left(R_{\mathrm{G}}\right) \propto R_{\mathrm{G}}^{-3.5}$ and $\rho_{b}\left(R_{\mathrm{G}}\right) \propto R_{\mathrm{G}}^{-4.0}$ are indicated by the solid and dashed lines, respectively. The curves have been arbitrarily scaled to match at $R_{\mathrm{G}}=2 \mathrm{kpc}$. (Lower panel) Radial distribution of globular clusters for the simulation shown in Fig. 4. The open histogram shows the initial profile, while the solid histogram indicates the distribution of the surviving globular clusters.

(Gunn \& Griffin 1979; Pryor \& Meylan 1993). In the second case, we have assumed $M / L_{V}=2$ and a universal dark halo mass of $M \simeq 2.0 \times 10^{7} M_{\odot}$, as suggested by studies of the internal kinematics Local Group dwarf galaxies (Mateo et al. 1993).

These simulations suggest that proto-Galactic spheroid was highly fragmented into numerous distinct, chemically isolated fragments. For $\alpha=-2$, the total number of fragments is $N_{\text {PGF }} \sim(1-2) \times 10^{3}$, which follows directly from the assumed power-law index for the luminosity function, the total luminosity of the Galactic spheroid, and the adopted faint-end cutoff of the proto-Galactic luminosity function. The majority of these proto-Galactic fragments are low-luminosity systems, with roughly $95 \%$ of the fragments having $L_{V} \lesssim 1.6 \times 10^{7} L_{V \text {, } \odot}$ (i.e., the present-day luminosity of the Fornax dSph galaxy). As a whole, these faint fragments contribute nearly half of the total halo luminosity but, by virtue of their low luminosity, none of its globular clusters. This is evident in the upper panel of Figure 5 which shows, for one simulation, the metallicities of halo stars and globular clusters plotted against the luminosity of the proto-Galactic fragment in which they originated. Although the globular clusters and field stars have similar mean metallicities, the field star distribution extends to both higher and lower metallicities, as evident in the lower panel of Figure 5.

Given the large number of proto-Galactic fragments required by hierarchical formation models to match the observed metallicity distributions, it is interesting to consider the possible implications for the mass budget of the Galaxy. If it is assumed that each fragment consists of a luminous component having $M / L_{V}=2$ which is embedded in a constant mass dark matter halo as described above, then for the adopted cutoff of $L_{V}=2.7 \times 10^{5} L_{V, \odot}$, the total mass is $M \simeq 3 \times 10^{10} M_{\odot}$. This is much lower than the total Galactic mass of $M \sim(3-9) \times 10^{11} M_{\odot}$ (Zaritsky et al. 1989; Kochanek 1996), suggesting that the protoGalactic fragments alone cannot account for the dark matter content of the Milky Way.

\subsection{A Second Example: The M31 Spheroid}

M31 has traditionally presented a challenge to models of halo formation since it is difficult to understand why its halo stars are, on average, 4 times more metal-rich than its globular clusters (Mould \& Kristian 1986; Brodie \& Huchra 1991). This difference is all the more puzzling in light of the fact that these components in the Milky Way - the other large Local Group spiral-have nearly identical mean metallicities.

A possible explanation of this difference is shown in Figure 8. In the upper panel, we compare the observed and simulated globular cluster metallicity distribution for M31; the lower panel shows the observed and simulated metallicity distributions for M31 halo stars. The data are taken from Barmby et al. (2000) and Holland, Richer, \& Fahlman (1996), respectively. No attempt has been made to include dynamical effects for the globular clusters since there are no published calculations of the dynamical evolution of the M31 globular cluster system. While dynamical effects will influence the relative numbers of globular clusters in the metal-rich and metal-poor populations, the mean metallicities of the two components will be not be affected. Following Walterbos \& Kennicutt (1988), we adopt $L_{V}^{s}=9.8$ $\times 10^{9} L_{V, \odot}$ for M31 and assume an identical bulge-to-halo ratio as for the Milky Way. Experiments indicate that $\alpha \sim-1.8$ most frequently produces the best match to the observed halo star distribution, particularly the peak at $[\mathrm{Fe} / \mathrm{H}] \simeq-0.7$. The observed and simulated cluster distributions, meanwhile, have their maxima at $[\mathrm{Fe} / \mathrm{H}] \simeq$ -1.3 owing to the fact that the assumed luminosity function of the proto-Galactic fragments is slightly more skewed toward higher luminosity fragments than was the case for the Milky Way. On the other hand, the prominent metalpoor tail seen in the halo star distribution is less pronounced in the simulations. As Holland et al. (1996) point out, the significance of this tail is unclear since confusion between metal-poor red giant branch stars and metal-rich asymptotic giant branch stars becomes important at this level.

Figure 9 shows the dependence of halo star and globular cluster metallicity on proto-Galactic fragment luminosity. A comparison with Figure 7 reveals the clear differences between the simulated metallicity distribution for the Galaxy and M31. In the case of M31, the proto-Galactic fragments have a slightly flatter luminosity function which results in a field star distribution whose mean metallicity is several times higher than that of the associated globular clusters.

\section{DISCUSSION AND IMPLICATIONS}

The simulations presented here demonstrate that hierarchical models are able to provide an excellent match to the metallicity distributions of Galactic globular clusters and halo field stars, provided the luminosity function of protoGalactic fragments had the form $d N \propto L^{\alpha} d L$ with $\alpha \sim-2$. Such a steep slope is in agreement with the predictions of semianalytic/numerical models of hierarchical galaxy for- 

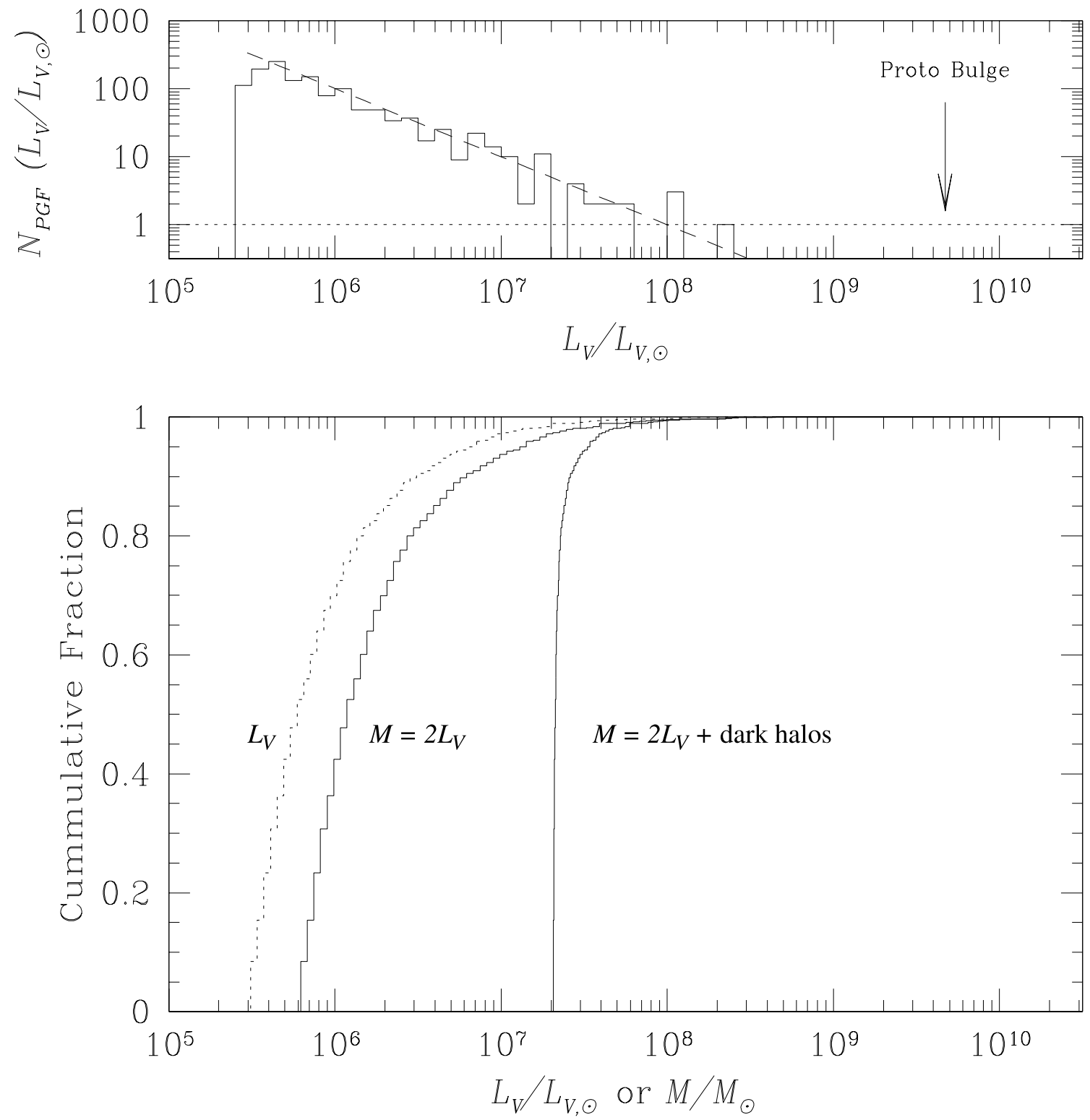

FIG. 7.-(Upper panel) Luminosity distribution proto-Galactic fragments for the simulation shown in Fig. 4. A power-law luminosity function with slope $\alpha=-2$ is shown as the dashed line. The location of the "protobulge" is indicated by the arrow. (Lower panel) Cumulative distribution of proto-Galactic fragment luminosities for the same simulation (dotted curve). The two solid curves show the cumulative distribution of proto-Galactic fragment masses assuming (1) $M=2 L_{V}\left(M_{\odot} / L_{V, \odot}\right)$ and (2) $M=2 L_{V}\left(M_{\odot} / L_{V, \odot}\right)+2 \times 10^{7} M_{\odot}$.

mation and the standard assumptions regarding gas cooling in dark halos (e.g., White \& Rees 1978; Blumenthal et al. 1984; Kauffmann et al. 1993; Klypin et al. 1999; Moore et al. 1999). It is, however, strongly inconsistent with the presently observed luminosity function of the Local Group and of the field galaxy population in general. While it is undoubtedly true that the current census of Local Group galaxies is incomplete at the faint end, it is highly unlikely that the number of Local Group dwarf galaxies has been underestimated by more than an order of magnitude. For instance, Pritchet \& van den Bergh (1999) have shown that the Local Group luminosity function closely resembles a Schechter function having $\alpha \simeq-1.1$ and that, based on the current census of Local Group galaxies, the probability of $\alpha<-1.3$ for a single Schechter function is less than $1 \%$.

Is it possible to reconcile these results? It is worth pointing out that this discrepancy is not unique to the simulations presented here but is, rather, a long-standing problem for semianalytic and numerical hierarchical models. For instance, Klypin et al. (1999) noted, on the basis of highresolution cosmological simulations of the Local Group, that the number of low-mass, dark halos predicted by the models exceeds the observed number of faint galaxies by nearly an order of magnitude. The magnitude of the discrepancy is slightly less than the one found here, since Klypin et al. (1999) predicted $\sim 300$ halos within $1.5 \mathrm{Mpc}$ of the Local Group (compared to the observed number of $\sim 40$ satellites), although the larger number of protoGalactic fragments found in the present case may be a consequence of the different mass/luminosity cutoffs. That is to say, the simulations of Klypin et al. (1999) become incomplete below $v_{c} \sim 20 \mathrm{~km} \mathrm{~s}^{-1}$, whereas such low-mass systems are explicitly included in our Monte Carlo approach: i.e., $v_{c} \sim 10 \mathrm{~km} \mathrm{~s}^{-1}$ at the faint end of the proto-Galactic luminosity function (Mateo 1998).

We suggest that the proto-Galactic fragments discussed 


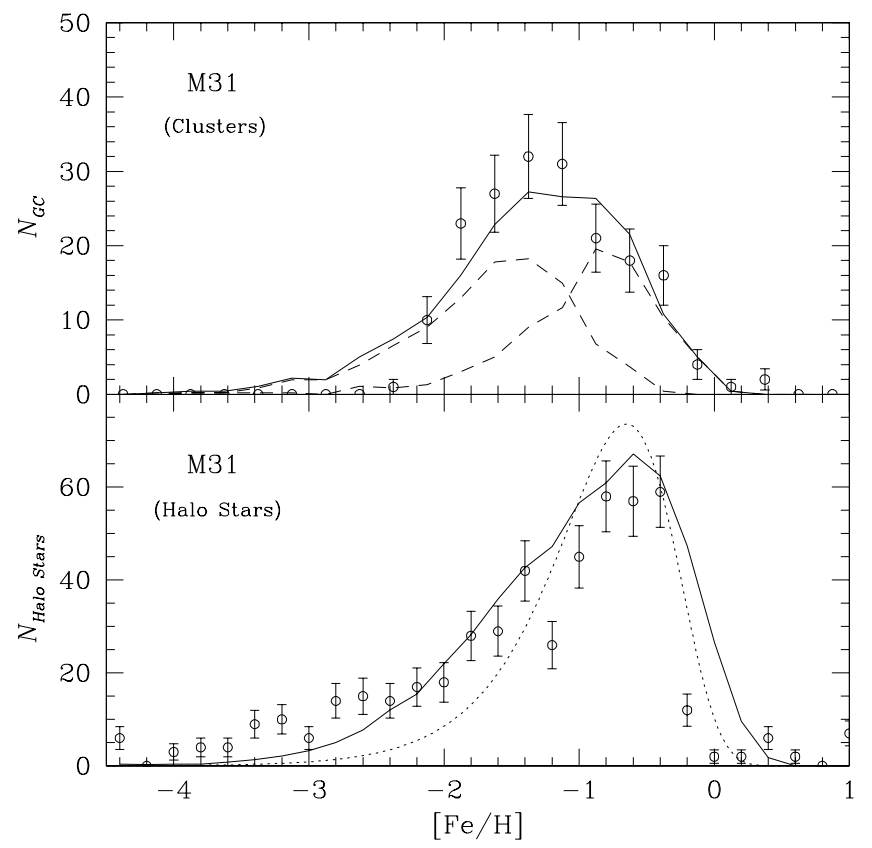

FIG. 8.-One simulation of the M31 globular cluster metallicity distribution. Dynamical effects have not been included. The separate metalrich and metal-poor components are indicated by dashed curves; the combined distribution is indicated by the solid curve. The open circles show the actual distribution using the cluster sample of Huchra, Brodie, \& Kent (1991). (Lower panel) Corresponding distribution of halo field star metallicities based on this simulation (solid curve). The halo star metallicities come from the G312 field of Holland, Richer, \& Fahlman (1996). The dotted line shows the prediction of the Simple Model for an effective yield of $\log y_{e}=-0.65$.

here are plausible candidates for the low-mass, dark halos seen in the semianalytic and numerical models. The issue, however, is complicated by the fact that, in the present case, the spheroid is identified with the disrupted stellar components of these fragments, whereas $N$-body simulations suggest that low-mass halos are relatively immune to the destructive effects of the Galactic tidal field. If this association is correct, then some physical mechanism is required to erase the dark matter substructure observed in the numerical models, such as tidal heating of halos on predominately radial orbits (Moore et al. 1996; van den Bosch et al. 1999) or impulsive heating during rapid, halo-halo encounters (Moore et al. 1996).

Two possible explanations for the discrepancy between the predicted and observed luminosity functions were discussed by Klypin et al. (1999): high-velocity clouds (HVCs) and dark satellites. In the first case, the numerous HVCs which populate the Local Group are assumed to represent the observable counterparts of the lowest mass dark halos (Blitz et al. 1999). Several properties of the HVCs, such as their large numbers (about 2500 in the Local Group; Stark et al. 1992), their low masses (typically $3 \times 10^{7} M_{\odot}$ of neutral gas and roughly 10 times this amount of dark matter; Blitz et al. 1999), their presumed extragalactic nature, and their inferred high rate of accretion onto the Galaxy at early times (Blitz et al. 1999), make them attractive candidates for the proto-Galactic fragments described here. Evidence for spatial and kinematic connections between HVCs and at least some Local Group dwarf galaxies has recently been presented by Blitz \& Robishaw (1999) and Côté et al. (2000). However, the dSph and dE galaxies which we have identified as the surviving protoGalactic fragments have clearly managed to convert much of their initial gas reservoir into stars, something which is not true for the majority of the HVCs.

Klypin et al. (1999) also examined the possibility that many low-mass dark halos rapidly lost their gas owing to supernovae-driven winds or an intergalactic photoionizing background. Although these processes may be important for explaining the excess in the predicted number of dark halos over the number observed, they cannot resolve the discrepancy found here. If the hierarchical models are correct, then the very existence of the Galactic spheroid demonstrates that its constituent proto-Galactic fragments managed to form significant numbers of stars.

A related possibility is that the luminosity function of proto-Galactic fragments depends sensitively on local environment. The steep slopes required by hierarchical formation models in the immediate vicinity of the protoGalaxy might then be a consequence of pressure confinement in high-density regions (Babul \& Rees 1992), biased dwarf formation (West 1993; Ferguson \& Binggeli 1994), or some other mechanism which enhances the efficiency of gas cooling in low-mass halos. Indeed, the remarkable diversity in the star formation histories of Local Group dwarfs (e.g., Grebel 1999) provides prima facie evidence for the complexity of gas accretion, cooling, and ejection in these objects.

Finally, the simulations presented here provide no direct constraints on the timescale of spheroid assembly, but it is nevertheless possible to draw some general conclusions on the duration of spheroid assembly. While the existence of the disrupting Sagittarius dwarf galaxy (Ibata, Gilmore, \& Irwin 1995) suggests that the accretion process has continued up to the present day, other arguments indicate that the majority of proto-Galactic fragments must have been incorporated into the spheroid at very early times. First, the thinness of the Galactic disk, whose oldest stars are believed to be $10_{-1}^{+3}$ Gyr old (Wood \& Oswalt 1998; Knox, Hawkins, $\&$ Hambly 1999), may indicate that the number of massive satellites accreted over its lifetime has been small (Toth \& Ostriker 1992; Moore et al. 1999). ${ }^{5}$ Second, a majority of the Local Group $\mathrm{dSph} / \mathrm{dE}$ galaxies contain young and intermediate-age stellar populations, whereas the fraction of such stars in the halo is known to be small (i.e., $\lesssim 10 \%$; Unavane et al. 1996). However, it is important to bear in mind that the proto-Galactic fragments, if accreted and disrupted at early times, would not have had the opportunity to form stars over periods of time, as did the Local Group dwarfs. In summary, the available evidence seems to favor an early, and relatively rapid, timescale for the assembly of the Galactic spheroid.

\section{SUMMARY}

We have described a semiempirical technique for simulating the chemical evolution of the Galactic spheroid in hierarchical formation scenarios. The simulations include no gas dissipation but assume instead that the bulk of star and cluster formation occurred within distinct, chemically iso-

\footnotetext{
${ }^{5}$ Estimates of disk heating by infalling satellites are reduced if the disks are allowed to warp (e.g., Huang \& Carlberg 1997; Sellwood, Nelson, \& Tremaine 1998), but, as Moore et al. (1999) have pointed out, such a mechanism is unlikely to be effective in the present case owing to the large number of proto-Galactic fragments.
} 


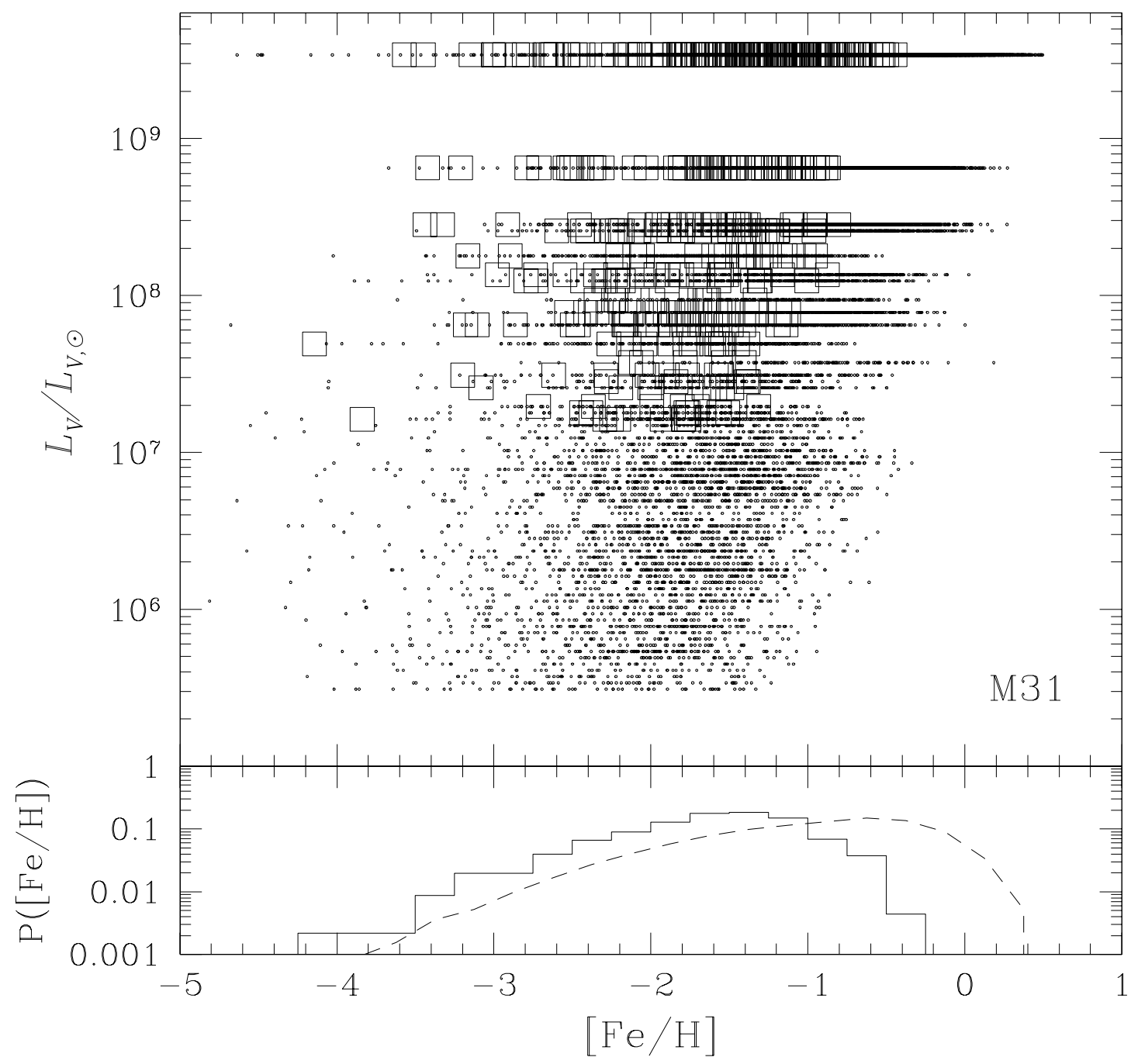

FIG. 9. - Same as Fig. 5, except for simulation of the M31 spheroid shown in the previous figure. Note that the M31 halo field star population is clearly more metal-rich than its globular cluster system owing to the smaller number of low-luminosity fragments incorporated into the spheroid.

lated proto-Galactic fragments which were subsequently assembled into the Galactic spheroid. The chemical enrichment of each proto-Galactic fragment is assumed to proceed in the manner predicted by the Simple Model (Searle \& Sargent 1972; Pagel \& Patchett 1975; Hartwick 1976). The effective yield of each fragment is determined empirically using the presently observed metallicityluminosity relations for stars and globular clusters belonging to nearby $\mathrm{dSph}$ and $\mathrm{dE}$ galaxies.

In this picture, the bulge is identified as the dominant proto-Galactic building block and the metal-rich Galactic globular clusters as its associated cluster system. This identification is supported by the observation that the metallicities of bulge field stars and the metal-rich Galactic globular clusters are consistent with the extrapolated metallicity-luminosity relations of dwarf galaxies (e.g., the smaller proto-Galactic fragments). By contrast, the Galactic halo is identified as the disrupted remains of numerous, much smaller, proto-Galactic fragments. A comparison between the observed and simulated metallicity distributions of Galactic globular clusters and halo field stars shows good agreement, provided the luminosity function of protoGalactic fragments has the form $d N \propto L^{\alpha} d L$, where $\alpha \sim-2$. When combined with the observed luminosity of the Galac- tic halo, this steep slope implies that the proto-Galactic spheroid was fragmented into $N_{\mathrm{PGF}} \sim 10^{3}$ distinct starforming regions; the metal-poor Galactic globular clusters formed in the approximately one dozen most massive fragments, whereas the bulk of the halo field star population and, in particular, the most metal-deficient objects, originated in numerous smaller fragments.

While these simulations provide independent support for semianalytic and numerical models of hierarchical galaxy formation, they exacerbate the long-standing discrepancy between the observed and predicted number of nearby faint galaxies.

We thank Pauline Barmby, Sean Ryan, and Steve Holland for providing the cluster and stellar metallicities shown in Figures 4 and 9. Thank also to the referee, Mario Mateo, for his many helpful suggestions. P. C. acknowledges support provided by the Sherman M. Fairchild Foundation. Additional support for this work was provided to R. O. M. by NASA through grant HF-0.096.01-97A from the Space Telescope Science Institute, which is operated by the Association of Universities for Research in Astronomy, Inc., under NASA contract NAS 5-26555. M. J. W. acknow- 
ledges financial support from the Natural Sciences and Engineering Research Council of Canada. D. M. is supported by the Chilean Fondecyt Project 01990440 and by the U. S. Department of Energy by Lawrence Livermore National Laboratory under contract W-7405-Eng-48. This research has made use of the NASA/IPAC Extragalactic Database (NED), which is operated by the Jet Propulsion Laboratory, California Institute of Technology, under contract with the National Aeronautics and Space Administration.
Aguilar, L., Hut, P., \& Ostriker, J. P. 1988, ApJ, 335, 720

Armandroff, T. E., \& Zinn, R. 1998, AJ, 96, 92

Ashman, K. A., \& Bird, C. M. 1993, AJ, 106, 2281

Ashman, K. A., \& Zepf, S. E. 1992, ApJ, 384, 50

Babul, A., \& Rees, M. J. 1992, MNRAS, 255, 346

Barbuy, B., Bica, E., \& Ortolani, S. 1998, A\&A, 333, 117

Barmby, P., Huchra, J. P., Brodies, J. P., Forbes, D. A., Schroder, L. L., \& Grillmair, C. J. 2000, AJ, 119, 727

Baugh, C. M., Cole, S., Frenk, C. S., \& Lacey, C. G. 1998, ApJ, 498, 504

Bender, R. Burstein, D., \& Faber, S. M. 1992, ApJ, 399, 462

Binney, J., \& Merrifield, M. 1998, Galactic Astronomy (Princeton: Princeton Univ. Press) 311

Blanchard, A., Valls-Gabaud, D., \& Mamon, G. A. 1992, A\&A, 264, 365

Blanco, V. M., \& Terndrup, D. M. 1989, AJ, 98,843

Blitz, L., \& Robishaw, T. 1999, preprint

Blitz, L., Spergel, D. N., Teuben, P. J., Hartmann, D., \& Butler, B. W. 1999, ApJ, 514, 818

Blumenthal, G. R., Faber, S. M., Primack, J. R., \& Rees, M. J. 1984, Nature, 311,517

Brodie, J. P., \& Huchra, J. P. 1991, ApJ, 379, 157

Caldwell, N., Armandroff, T. E., Seitzer, P., \& Da Costa, G. S. 1992, AJ, 103,840

Carney, B. W., Laird, J. B., Latham, D. W., \& Aguilar, L. A. 1996, AJ, 112, 668

Chen, B. 1998, ApJ, 495, L1

Cole, S., Aragón-Salamanca, A., Frenk, C. S., Navarro, J. F., Zepf, S. E. 1994, MNRAS, 271, 781

Côté, P. 1999, AJ, 118, 406

Côté, P., Marzke, R. O., \& West, M. J. 1998, ApJ, 501, 554

Côté, P., Oke, J. B., \& Cohen, J. G. 1999, AJ, 118, 1645

Côté, P., Sargent, W. L. W., Mateo, M., \& Olszewski, E. W. 2000, ApJL, submitted

Davies, R., Burstein, D., Dressler, A., Faber, S., Lynden-Bell, D., Terlevich, R., \& Wegner, G. 1987, ApJS, 64, 581

Dekel, A., \& Silk, J. 1986, ApJ, 303, 39

de Vaucouleurs, G., \& Pence, W. D. 1978, AJ, 83, 1163

Durrell, P. R., Harris, W. E., Geisler, D., \& Pudritz, R. E. 1996, AJ, 112, 972

Dwek, E., et al. 1995, ApJ, 445, 716

Eggen, O. J., Lynden-Bell, D., \& Sandage, A. R. 1962, ApJ, 136, 748

Fall, S. M., \& Rees, M. J. 1985, ApJ, 298, 18

Ferguson, H. C., \& Binggeli, B. 1994, A\&A Rev., 6, 67

Forbes, D. A., Brodie, J. P., \& Grillmair, C. J. 1997, AJ, 113, 1652

Frogel, J. A., Terndrup, D. M., Blanco, V. M., \& Whitford, A. E. 1990, ApJ, 353,494

Gebhardt, K., \& Kissler-Patig, M. 1999, AJ, 118, 1526

Geisler, D., Lee, M. G., \& Kim, E. 1996, AJ, 111, 1529

Gilmore, G., King, I. R., \& van der Kruit, P. C. 1990, The Milky Way as a

Galaxy (Mill Valley: University Science Books)

Gnedin, O. Y., \& Ostriker, J. P. 1997, ApJ, 474, 223

Grebel, E. K. 1999, in IAU Symp. 192, The Stellar Content of Local Group

Galaxies, ed. P. Whitelock \& R. Cannon (San Francisco: ASP), 17

Gunn, J. E., \& Gott, J. R. 1972, ApJ, 176, 1

Gunn, J. E., \& Griffin, R. F. 1979, AJ, 84, 752

Haehnelt, M. G., Steinmetz, M., \& Rauch, M. 1998, ApJ, 495, 647

Harris, W. E. 1976, AJ, 81, 1095

1981, ApJ, 251, 497

1986, AJ, 91, 822

1991, ARA\&A, 29, 543

1996, AJ, 112, 1487

Harris, W. E., \& Pudritz, R. E. 1994, ApJ, 429, 177

Harris, W. E., \& van den Bergh, S. 1981, AJ, 86, 1627

Hartwick, F. D. A. 1976, ApJ, 209, 418

Hesser, J. E., et al. 1998, in IAU Symp. 192, The Stellar Content of Local

Group Galaxies, ed. P. Whitelock \& R. Cannon (San Francisco: ASP), 4

Holland, S., Richer, H. B., \& Fahlman, G. G. 1996, AJ, 112, 1035

Holtzman, J. A., et al. 1998, AJ, 115, 1946

Huang, S., \& Carlberg, R. G. 1997, ApJ, 480, 503

Huchra, J. P., Brodie, J. P., \& Kent, S. M. 1991, ApJ, 370, 495

Ibata, R., Gilmore, G., \& Irwin, M. 1994, Nature, 370, 194 1995, MNRAS, 277, 781

Jablonka, P., Martin, P., \& Arimoto, N. 1996, AJ, 112, 1415

Kauffmann, G., White, S. D. M., \& Guiderdoni, B. 1993, MNRAS, 264, 201

King, J. R. 1997, AJ, 113, 2302

Kinman, T. D. 1959, MNRAS, 119, 538

Kinman, T. D., Pier, J. R., Suntzeff, N. B., Harmer, D. L., Valdes, F.,

Hanson, R. B., Klemola, A. R., \& Kraft, R. P. 1996, AJ, 111, 1164

Kissler-Patig, M. 1997, A\&A, 319, 83
Kissler-Patig, M., Ashman, K., Zepf, S. E., \& Freeman, K. C. 1999, AJ, 118, 197

Kissler-Patig, M., Kohle, S., Hilker, M., Richtler, T., Infante, L., \& Quintana, H. 1997b, A\&A, 319, 470

Kissler-Patig, M., Richtler, T., Storm, J., \& Della Valle, M. 1997b, A\&A 327,503

Klypin, A. A., Kravtsov, A. V., Valenzuela, O., \& Prada, F. 1999, ApJ, 522,

Knox, R. A., Hawkins, M. R. S., \& Hambly, N. C. 1999, MNRAS, 306, 736

Kochanek, C. S. 1996, ApJ, 457, 228

Kormendy, J. 1985, ApJ, 295, 73

Kormendy, J., \& Illingworth, G. 1983, ApJ, 265, 632

Kundu, A. 1999, Ph.D. thesis, Univ. Maryland

Kundu, A., \& Whitmore, B. C. 1998, AJ, 116, 2841

Laird, J. B., Carney, B. W., Rupen, M. P., \& Latham, D. W. 1988, AJ, 96, 1908

Laird, J. B., \& Sneden, C. 1996, in ASP Conf. Ser. 92, Formation of the Galactic Halo ... Inside and Out, ed. H. Morrison \& A. Sarajedini (San Francisco: ASP), 192

Larson, R. B. 1988, in IAU Symp. 126, Globular Cluster Systems in Galaxies, ed. G. Grindlay \& A. G. D. Philip (Dordrecht: Reidel), 311

Lee, M. G., Kim, E., \& Geisler, D. 1998, AJ, 115, 947

Majewski, S. R., Munn, J. A., \& Hawley, S. L. 1996, ApJ, 459, L73

Marzke, R. O., Da Costa, L. N., Pellegrini, P. S., Willmer, C. N. A., \& Geller, M. J. 1998, ApJ, 503, 617

Mateo, M. 1996, in ASP Conf. Ser. 92, Formation of the Galactic Halo ... Inside and Out, ed. H. Morrison \& A. Sarajedini (San Francisco: ASP), 434

Mateo, M. 1998, ARA\&A, 36, 435

Mateo, M., Olszewski, E. W., Pryor, C., Welch, D. L., \& Fischer, P. 1993 , AJ, 105, 510

McLaughlin, D. E. 1999, AJ, 117, 2398

McWilliam, A., \& Rich, R. M. 1994, ApJS, 91, 749

Miller, B. W., Lotz, J. M., Ferguson, H. C., Stiavelli, M., \& Whitmore, B. C. 1998, ApJ, 508, L133

Minniti, D. 1995, AJ, 109, 1663

Minniti, D., Olszewski, E. W., Liebert, J., White, S. D. M., Hill, J. M., \& Irwin, M. J. 1995, MNRAS, 277, 1293

Moore, B., Ghigna, S., Governato, F., Lake, G., Quinn, T., Stadel, J., \& Tozzi, P. 1999, ApJ, 524, L19

Moore, B., Katz, N., \& Lake, G. 1996, ApJ, 457, 455

Morgan, W. W. 1959, AJ, 64, 432

Morrison, H. L. 1996, in ASP Conf. Ser. 92, Formation of the Galactic Halo ... Inside and Out, ed. H. Morrison \& A. Sarajedini (San Francisco: ASP), 453

Mould, J. R., \& Kristian, J. 1986, ApJ, 305, 591

Murali, C., \& Weinberg, M. D. 1997, MNRAS, 291, 717

Neilsen, E. H. 1999, in Ph.D. thesis, Johns Hopkins Univ.

Neilsen, E. H., \& Tsvetanov, Z. I. 1999, ApJ, 515, L13

Ostriker, J. P., Spitzer, L., \& Chevalier, R. 1972, ApJ, 176, L51

Ostrov, P., Geisler, D. \& Forte, J. C. 1993, AJ, 105, 1762

Pagel, B. E. J., \& Patchett, B. E. 1975, MNRAS, 172, 13

Press, W. H., \& Schechter, P. 1974, ApJ, 187, 425

Preston, G. W., Beers, T. C., \& Schectman, S. 1994, AJ, 108, 538

Preston, G. W., Schectman, S., \& Beers, T. C. 1991, ApJ, 375, 121

Pritchet, C. J., \& van den Bergh, S. 1999, AJ, 118, 883

Pryor, C., \& Meylan, G. 1993, in ASP Conf. Ser. 50, The Structure and Dynamics of Globular Clusters, ed. S. G. Djorgovski \& G. Meylan (San Francisco: ASP), 357

Renzini, A. 2000, in The Formation of Galactic Bulges, ed. M. Carollo, H. C. Ferguson, \& R. F. G. Wyse (Cambridge: Cambridge Univ. Press), in press

Ryan, S. G., \& Norris, J. N. 1991, AJ, 101, 1865

Saha, A. 1985, ApJ, 289, 310

Sandage, A. R. 1990, JRASC, 84, 70

Sarajedini, A., Chaboyer, B., \& Demarque, P. 1997, PASP, 109, 1321

Schechter, P. 1976, ApJ, 203, 297

Schweizer, F. 1986, in Nearly Normal Galaxies, ed. S. Faber (New York: Springer), 18

Searle, L., \& Sargent, W. L. W. 1972, ApJ, 173, 25

Searle, L., \& Zinn, R. 1978, ApJ, 225, 357

Sellwood, J. A., Nelson, R. W., \& Tremaine, S. 1998, ApJ, 506, 590

Simien, F., \& de Vaucouleurs, G. 1986, ApJ, 302, 564

Stark, A. A., Gammie, C. F., Wilson, R. W., Bally, J., Linke, R. A., Heiles, C., \& Hurwitz, M. 1992, ApJS, 79, 77

Steinmetz, M., \& Müller, E. 1995, MNRAS, 276, 549

Suntzeff, N. B., Kinman, T. D., \& Kraft, R. P. 1991, ApJ, 367, 528 
Terndrup, D. M. 1988, AJ, 96, 884

Toth, G., \& Ostriker, J. P. 1992, ApJ, 389, 5

Tremaine, S. D. 1976, ApJ, 203, 345

Tully, R. B. 1988, Nearby Galaxies Catalog (Cambridge: Cambridge Univ. Press)

Unavane, M., Wyse, R. F. G., \& Gilmore, G. 1996, MNRAS, 278, 727

van den Bergh, S. 1982, PASP, 94, 459

- 1996, PASP, 108, 986

van den Bosch, F. C., Lewis, G. F., Lake, G., \& Stadel, J. 1999, ApJ, 515, 50

Walterbos, R. A. M., \& Kennicutt, R. C. 1988, A\&A, 198, 61
West, M. J. 1993, MNRAS, 265, 755

White, S. D. M., \& Rees, M. J. 1978, MNRAS, 183, 341

Whitmore, B. C., Sparks, W. B., Lucas, R. A., Macchetto, F. D., \& Biretta, J. A. 1995, ApJ, 454, L73

Wood, M. A., \& Oswalt, T. D. 1998, ApJ, 497, 870

Zaritsky, D., Olszewski, E. W., Schommer, R. A., Peterson, R. C., \& Aaronson, M. 1989, ApJ, 345, 759

Zinn, R. 1985, ApJ, 293, 424

. 1993, in ASP Conf. Ser. 48, The Globular Cluster-Galaxy Connection, ed. G. Smith \& J. Brodie (San Francisco: ASP), 603 